\title{
Der Bundestag im Spannungsfeld von politischer Partizipation und wissenschaftlicher Expertise
}

\author{
Brown, Mark B., Justus Lentsch und Peter Weingart: Politikberatung und Parlament, Verlag \\ Barbara Budrich, Opladen / Farmington Hills 2006, 174 Seiten, € 22,-.
}

Die durch die gesellschaftliche Pluralisierung und Entstrukturierung vervielfachten Interessengegensätze und die Entstehung neuartiger Problemlagen wie Globalisierung oder Klimawandel reduzieren die Möglichkeiten sozialer Repräsentation und konventioneller parlamentarischer Interessenvertretung. Stattdessen ist im zunehmenden Maße die politische Integrationsleistung von Parlamenten gefordert. Repräsentation ist nicht mehr in erster Linie Vertretung, sondern zuerst Gestaltung - so das zugespitzte Fazit von Studien zum Funktionswandel moderner Parlamente. Solche parlamentarische Gestaltung bedarf einer intensiven Kommunikation mit der Gesellschaft, einerseits um über die sich wandelnden Problemlagen und Ansprüche von Wählern und gesellschaftlichen Gruppen informiert zu sein, andererseits um im Geflecht politischer Institutionen zur Problemlösung beitragen zu können.

In den letzten Jahren haben sich verschiedene Studien mit einzelnen Aspekten dieses Kommunikationsprozesses zwischen Parlament und Gesellschaft beschäftigt, seien es Arbeiten zu den Netzwerken von Abgeordneten oder zum Einfluss von Lobbyisten. Die Rolle der wissenschaftlichen Politikberatung fand dabei bislang seltener Beachtung. Dazu haben nun Mark Brown, Justus Lentsch und Peter Weingart ein schmales, aber informiertes und informatives Buch vorgelegt, das auf einem Gutachten für das Büro für Technikfolgenabschätzung beim Deutschen Bundestag beruht. Um die wissenschaftliche Politikberatung von nichtwissenschaftlichen Formen der Politikberatung, etwa durch Nichtregierungsorganisationen, Stiftungen oder Lobbyisten, abzugrenzen, bestimmen die Autoren drei Kriterien. Dezidiert wissenschaftliche Politikberatung sei eine institutionalisierte Informationslieferung, die sich (a) an politische Entscheidungsträger richtet, (b) aus einer methodologischen Systematik entstanden ist und (c) auf wissenschaftlichem Wissen beruht (S. 16). Man kann darüber streiten, ob diese Definition tatsächlich die Beratung durch Verbände oder wissenschaftliche Mitarbeiter von Abgeordneten, wie es die Autoren beabsichtigen, ausschließt.

Den Kern des Buches bilden drei Fallstudien, anhand derer der Beitrag der wissenschaftlichen Politikberatung zu den Aufgaben des Bundestages untersucht wird. Betrachtet werden Enquete-Kommissionen, das Büro für Technikfolgenabschätzung (TAB) und partizipatorische Gremien wie Planungszellen oder Konsensuskonferenzen. Diese drei Formen der Politikberatung werden aus zwei Perspektiven bewertet: Repräsentativität und Resonanz. Gefragt wird also, in welchem Maße die Gremien bei der Mitgliederauswahl, der Themensetzung und in ihrer Verfahrensweise die jeweils relevanten unterschiedlichen fachlichen, sozialen und politischen Perspektiven berücksichtigen und in welchen Umfang die Beratungsergebnisse im parlamentarischen Diskurs aufgenommen werden.

Der empirischen Studie über die Beratungsressourcen des Bundestages werden ausführliche theoretische Reflexionen zur politischen Repräsentation und zu den Merkmalen wie Dilemmata der Politikberatung vorangestellt. Die Autoren folgen einem Repräsentationsverständnis, das an die Theorie von Hanna F. Pitkin anknüpft und sich auf die Arbeiten von Dietrich Herzog bezieht. Repräsentation ist danach nicht als direkte Beziehung zwi- 
schen Volksvertretern und Wählern zu verstehen, sondern als komplexer Interaktionsprozess zwischen dem Parlament und diversen Institutionen, Organisationen und Akteuren (S. 37), an dem auch wissenschaftliche Politikberatung mitwirkt. Das Parlament steht vor der Aufgabe, zwischen zwei verschiedenen Legitimationsressourcen zu vermitteln - Legitimation durch Wahl sowie durch Bezug auf wissenschaftliche Beratung, um die Rationalität politischer Entscheidungen zu sichern. Dabei ist das Verhältnis von Wissenschaft und Politik vielschichtig: Zum einen ist Expertenwissen zur Identifizierung und Lösung politischer Probleme notwendig. Zum anderen kann es, unabhängig vom tatsächlichen Beitrag zur Problemlösung, dazu dienen, politischen Entscheidungen öffentliche Akzeptanz zu verschaffen, weil Experten vermeintlich dem Parteienstreit entzogen sind. Es kann aber auch delegitimierend wirken, wenn sich wissenschaftliche Analysen als falsch beziehungsweise überlebt erweisen oder der Eindruck einer technokratischen Herrschaft entsteht, der die öffentliche Zustimmung fehlt. Die Autoren fordern daher, nach Wegen zu suchen, die „Partizipation mit politischer Repräsentation und diese wiederum mit wissenschaftlicher Politikberatung sinnvoll verbinden" (S. 48). Als ein Weg wird die Demokratisierung von Expertise diskutiert, also die Beteiligung von Laien am Beratungsprozess, um den Experteneinfluss zurückzudrängen. Bereits hier wird die Sympathie der Autoren für so genannte Bürgerkonferenzen sichtbar, deren Stärkung auch in der abschließenden Formulierung von Reformempfehlungen vorgeschlagen wird.

Der empirische Teil des Buches beginnt mit einem Überblick über die Entwicklung der wissenschaftlichen Politikberatung des Bundestages; anschließend werden jeweils die Geschichte und die institutionellen Eigenarten von Enquete-Kommissionen, des TAB und von Bürgerkonferenzen beschrieben. Alle drei Formen der Politikberatung werden hinsichtlich ihrer Repräsentativität sowie ihrer parlamentarischen und öffentlichen Resonanz analysiert. Die Bürgerkonferenzen unterscheiden sich von den beiden übrigen Formen darin, dass sie bislang nicht im Umfeld des Bundestags stattgefunden haben. Relevante Erfahrungen mit Bürgerkonferenzen gibt es in Deutschland nur auf der kommunalen Ebene sowie in Dänemark und den Niederlanden bei den Nationalparlamenten. Im parlamentarischen System Deutschlands stellen sie also nur eine potenzielle Beratungsquelle dar.

Vor dem Hintergrund des theoretischen Rahmens überraschen die Ergebnisse der empirischen Analyse kaum: Das TAB zeichnet sich durch hohe fachliche Repräsentativität und geringe politische und soziale Repräsentativität aus. Es genießt eine hohe Autonomie. Weil die Besetzung der Enquete-Kommissionen durch die Fraktionen erfolgt, ist ihre politische und soziale Repräsentativität größer, ohne die fachliche Repräsentativität zu vernachlässigen. Schließlich haben sie einen unter öffentlicher Beobachtung stehenden Untersuchungsauftrag, der in einem nur nach Proporzgesichtspunkten besetzten Gremium schwer zu erfüllen wäre. Am stärksten betonen Bürgerkonferenzen sowohl die fachliche als auch die soziale Repräsentativität. Die größte parlamentarische Resonanz haben das TAB, die geringste Bürgerkonferenzen. Dafür ist die öffentliche Resonanz der Bürgerkonferenzen hoch. Enquete-Kommissionen werden sowohl innerparlamentarisch als auch öffentlich beachtet. Entsprechend unterscheiden sich die Funktionen, welche die verschiedenen Formen der wissenschaftlichen Politikberatung erfüllen: Während die Stärke von Bürgerkonferenzen in Artikulation, Integration und Beförderung des öffentlichen Verständnisses von Problemen besteht, ist das TAB leistungsstark bei der Identifizierung von Problemen und Handlungsoptionen. Enquete-Kommissionen zeichnet aus, dass sie die meisten politik- und problembezogenen Funktionen relativ gut erfüllen. 
Trotz einiger Redundanzen im theoretischen Teil überzeugt der Band sowohl hinsichtlich der theoretischen Einordnung der Formen von Politikberatung als auch in der empirischen Analyse. Bezweifelt werden darf aber, ob die Autoren mit ihren eigenen Beratungsvorschlägen Erfolg haben werden: Eine verstärkte Verbindung zwischen Parlament und Bürgerkonferenzen ist in Deutschland nicht absehbar.

Helmar Schöne

\title{
Verfassungsrechtliche Strukturen direkter Demokratie: dogmatisch starke Klärung
}

\author{
Hartmann, Bernd J.: Volksgesetzgebung und Grundrechte (Münsterische Beiträge zur Rechtswis- \\ senschaft, Bd. 160), Verlag Duncker \& Humblot, Berlin 2005, 248 Seiten, € 69, 80.
}

Angeregt zu seiner Untersuchung hat Bernd J. Hartmann ein Beschluss des Bundesverfassungsgerichts von 1997, der die Verfassungsbeschwerde der Beauftragten des bayerischen Volksbegehrens „Das bessere Müllkonzept“, die sich gegen unfaire Abstimmungspropaganda staatlicher und kommunaler Amtsträger wehrte, als unzulässig verwarf. Aber seine Studie malt nicht ein farbiges Panorama vergleichbarer Fälle und ähnlicher Judikate, sondern Hartmann möchte die vom Bundesverfassungsgericht entwickelte Lösung überprüfen, und dafür geht er das Problem prinzipiell, fast möchte man sagen: fundamental, an, als Frage nach der Grundrechtsgeltung im Volksgesetzgebungsverfahren: Wann lebt der (gesetzgebende) Bürger seine grundrechtliche Freiheit aus, und wann ist der (bürgerliche) Gesetzgeber, da er Staatsgewalt ausübt, grundrechtsgebunden? Seine „an Grundsätzlichem interessierte und zum Grundgesetz geschriebene Abhandlung" (S. 132) abstrahiert konsequenterweise von den Landesverfassungen und der Staatspraxis in den Ländern (sowie erst recht von den Normen und der Wirklichkeit von Bürgerbegehren und Bürgerentscheid nach Kommunalverfassungsrecht), was insofern nicht ohne Pointe ist, als es Hartmann nicht nur um eine eventuell einzuführende Bundesvolksgesetzgebung geht - die zu der Zeit, als er seine Studie erarbeitete, in der rot-grünen Koalitionsvereinbarung stand -, sondern seine Auslegung des Grundgesetzes selbstverständlich Gültigkeit für die direkte Demokratie auf Landes- und Kommunalebene beansprucht.

In gewissem Widerspruch zu dem Abstraktionsvorsatz breitet der Verfasser erst noch eine umfassende Synopse der rechtlichen Grundlagen direktdemokratischer Sachentscheidungen in Ländern und Kommunen aus (S. 29 bis 42). Dann aber enteilt er auf die Höhen des Verhältnisses von Gesellschaft und Staat, von privatem und öffentlichem Recht, von subjektivem Recht und Kompetenz sowie von Grundrechtsberechtigung und Grundrechtsbindung. Für die Antwort auf seine fundamentale Frage arbeitet der Autor die Konturen des Modells eines grundgesetzlichen Volksgesetzgebungsverfahrens heraus, dessen einzelne Schritte er als grundrechtsberechtigten Freiheitsgebrauch (so zum Beispiel Entwurfsfertigung und Abstimmungskampf - letzteren im Gegensatz zu der eingangs erwähnten Entscheidung des Bundesverfassungsgerichts, vgl. S. 119) oder als grundrechtsgebundene Wahrnehmung staatlicher Kompetenz (so zum Beispiel Volksbegehren und Volksentscheid) einordnet. Das Grundgesetz enthält für dieses Unterfangen bekanntlich nur wenige Vor- 